\title{
Results from LHCb in charmless $b$-baryon decays
}

\author{
Andrea Merli ${ }^{* \dagger}$ \\ Università degli Studi di Milano and INFN-Milano, Milano \\ E-mail: andrea.merli@cern.ch
}

Since mostly unexplored, the heavy baryon sector is an interesting field where to search for new physics effects. Recent LHCb results in $b$-baryon decays to charmless final states are presented based on $p p$ collision data collected in 2011 and 2012 at centre-of-mass energies of $\sqrt{s}=7 \mathrm{TeV}$ and $8 \mathrm{TeV}$, respectively. The total integrated luminosity collected is $3.0 \mathrm{fb}^{-1}$. New decays of $b$-baryons are presented and preliminary studies of $C P$ violation are performed. The first evidence of $C P$ violation in baryons is provided in $\Lambda_{b}^{0} \rightarrow p \pi^{-} \pi^{+} \pi^{-}$decays by means of the triple-product correlation technique. All the other presented $C P$ asymmetries are compatible with zero.

9th International Workshop on the CKM Unitarity Triangle

28 November - 3 December 2016

Tata Institute for Fundamental Research (TIFR), Mumbai, India

${ }^{*}$ Speaker.
†n behalf of the LHCb collaboration. 


\section{Introduction}

The "B-factories" BaBar and Belle have studied in detail the $B^{0}$ and $B^{ \pm}$decays, however the heavy baryons sector (i.e. containing the $b$ quark) still remains largely unexplored. Given the copious production of heavy baryons at the LHC, precision measurements have become possible in this field and the interest of scientific community is growing on this subject. Recently, studies of heavy baryons led to the measurement of $\left|V_{u b}\right|$ using $\Lambda_{b}^{0} \rightarrow p \mu^{-} \bar{v}$ decays [1], to the discovery of pentaquark using $\Lambda_{b}^{0} \rightarrow J / \psi p K^{-}$decays [2] and also to the discoveries of $\Xi_{b}^{\prime}$ and $\Xi_{b}^{\prime *}$ baryonic states [3] and two orbitally excited $\Lambda_{b}^{* 0}$ states [4].

It is interesting to study baryon charmless decays, search new decay modes and measure their branching fraction, because in the Standard Model (SM) $C P$ violation (CPV) is predicted to be particularly suppressed, as explained by the CKM flavour mixing mechanism.A significant excess of CPV with respect to the theoretical predictions would represent a proof of new physics beyond the SM. Within the mesonic sector, the CKM matrix in the SM describes the CPV results for $B$ and $K$ mesons. It is important to test $\mathrm{CPV}$ also in baryon decays to verify if the mechanism through which it is generated is the same as for mesons. This is a complementary test of the SM with repect to what has been already done with mesons. Indeed both mesons and baryons are governed by the same underlying short distance physics, with different spin and QCD structure, and it is interesting to compare the two results.

Four recent results from $\mathrm{LHCb}$ in the field of charmless $b$-hadron decays are here reviewed. All the analysis are performed on the full Run I datasample corresponding to an integrated luminosity of $3.0 \mathrm{fb}^{-1}$.

\section{Study of $\Lambda_{b}^{0}\left(\Xi_{b}^{0}\right) \rightarrow \Lambda h^{+} h^{\left({ }^{\prime}\right)-}$ decays}

An inclusive measurement of $\Lambda_{b}^{0} \rightarrow \Lambda h^{+} h^{\left({ }^{\prime}\right)-}$ decays, where $h, h^{\prime} \in\{K, \pi\}$, has been performed [5]. The analysis uses the abundant $\Lambda_{b}^{0} \rightarrow \Lambda_{c}^{+} \pi$ control mode. The result of the maximum likelihood mass fit is shown in Figure 1. Signals for $\Lambda_{b}^{0} \rightarrow \Lambda K^{+} K^{-}$and $\Lambda_{b}^{0} \rightarrow \Lambda K^{+} \pi^{-}$are ob-
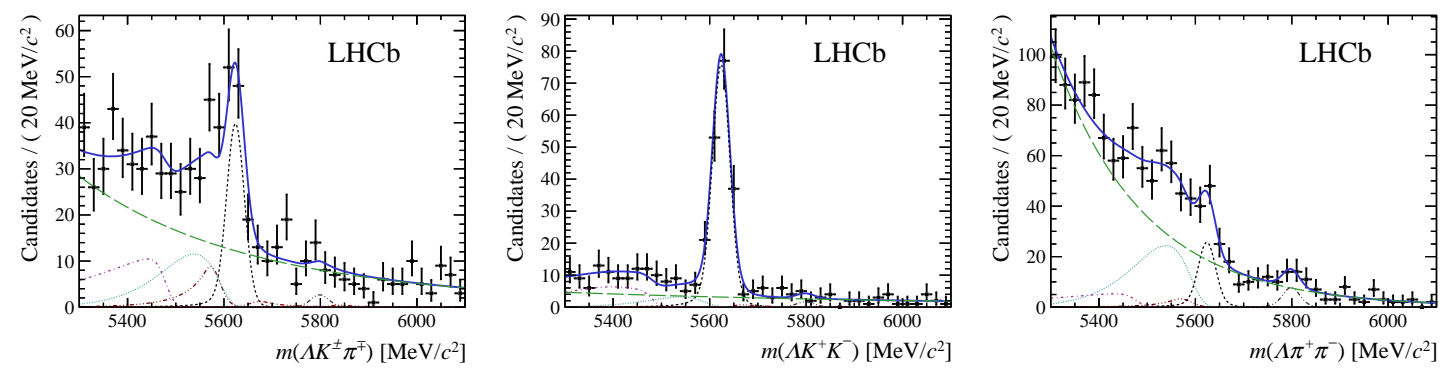

Figure 1: Results of the fit for the (left) $\Lambda K^{ \pm} \pi^{ \pm}$, (middle) $\Lambda K^{+} K^{-}$and (right) $\Lambda \pi^{+} \pi^{-}$final states. Superimposed on the data are the total result of the fit as a solid blue line, the $\Lambda_{b}^{0}\left(\Xi_{b}^{0}\right)$ signal contribution as a short-dashed black (double dot-dashed grey) line, cross-feed as triple dot-dashed brown lines, the combinatorial background as a long-dashed green line, and partially reconstructed background with either a missing neutral pion as a dot-dashed purple line or a missing soft photon as a dotted cyan line.

served with a significance of $15.8 \sigma$ and $8.1 \sigma$, respectively, where the systematic uncertainties 
are also taken into account. There is strong evidence for $\Lambda_{b}^{0} \rightarrow \Lambda \pi^{+} \pi^{-}(4.7 \sigma)$ while the significance of the other modes is less than $3 \sigma$. The branching fractions are measured to be $\mathscr{B}\left(\Lambda_{b}^{0} \rightarrow\right.$ $\left.\Lambda K^{+} \pi^{-}\right)=(5.6 \pm 0.8 \pm 0.8 \pm 0.7) \times 10^{-6}, \mathscr{B}\left(\Lambda_{b}^{0} \rightarrow \Lambda K^{+} K^{-}\right)=(15.9 \pm 1.2 \pm 1.2 \pm 2.0) \times 10^{-6}$ and $\mathscr{B}\left(\Lambda_{b}^{0} \rightarrow \Lambda \pi^{+} \pi^{-}\right)=(4.6 \pm 1.2 \pm 1.4 \pm 0.6) \times 10^{-6}$ where the first uncertainty is statistical, the second systematic and the third one is due to the precision with which the normalisation channel branching fraction is known. The significant yields observed in the $\Lambda_{b}^{0} \rightarrow \Lambda K^{+} K^{-}$and $\Lambda_{b}^{0} \rightarrow \Lambda K^{+} \pi^{-}$decays allows for the determination of phase-space-integrated $C P$ asymmetries. Those asymmetries are measured with respect to the control mode in order to cancel at first order the $\Lambda_{b}^{0}$ production and final state detection asymmetries. The $C P$ asymmetries are then found to be $\mathscr{A}_{C P}\left(\Lambda_{b}^{0} \rightarrow \Lambda K^{+} \pi^{-}\right)=-0.53 \pm 0.23 \pm 0.11$ and $\mathscr{A}_{C P}\left(\Lambda_{b}^{0} \rightarrow \Lambda K^{+} K^{-}\right)=-0.28 \pm 0.10 \pm 0.07$ where the uncertainties are statical and systematic, respectively. These are both consistent with zero, though will be interesting with larger dataset sizes.

\section{Study of $\Lambda_{b}^{0} \rightarrow \Lambda \phi$ decays}

In addition, a search for the exclusive mode $\Lambda_{b}^{0} \rightarrow \Lambda \phi$ is performed with LHCb data [6]. The approach is to fit in the three invariant mass dimensions $\left(M_{K^{+} K^{-}} p \pi^{-}, M_{K^{+} K^{-}}, M_{p \pi^{-}}\right)$depending if $\Lambda$ baryon decayed inside (long) or outside (downstream) the vertex locator. This then allows for the different selection efficiencies and resolutions to be accounted for. All the projections are shown in Figure 2. The mass fit results in a yield of $89 \pm 13$. The signal significance is
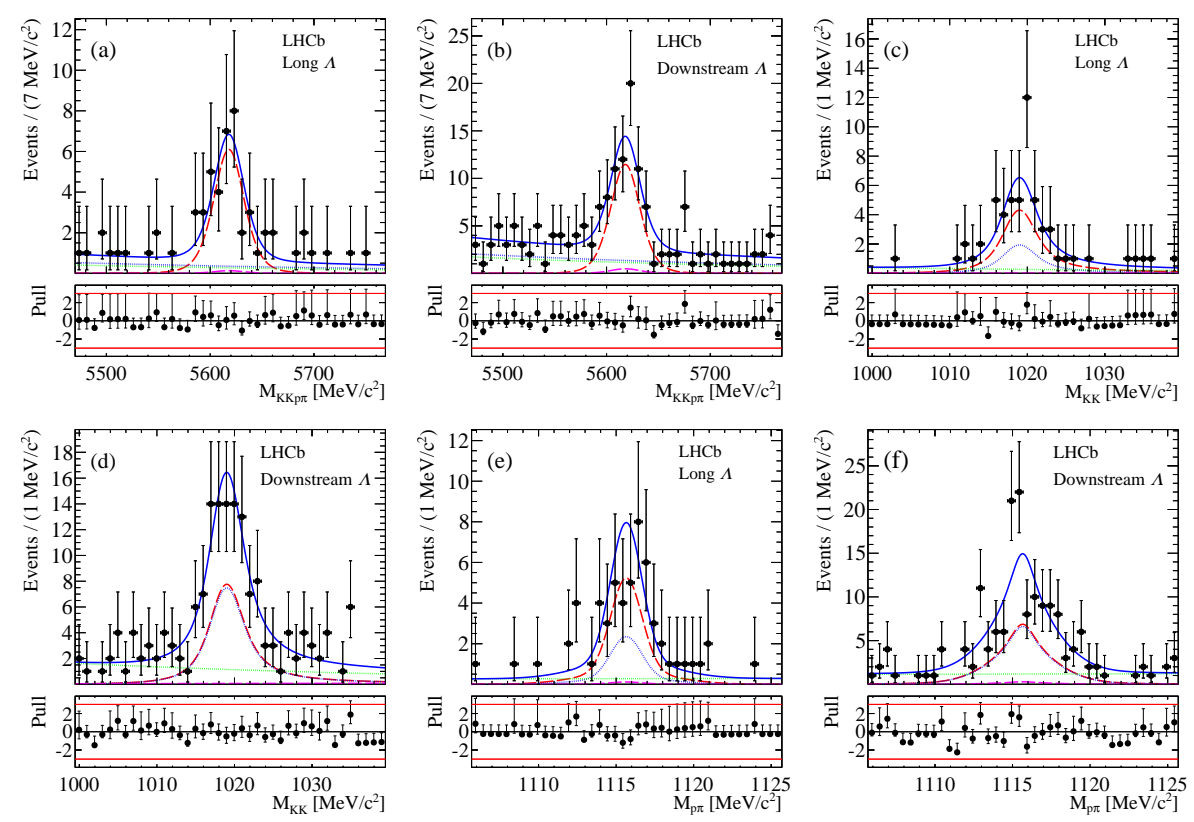

Figure 2: Fit projections to the $K^{+} K^{-} p \pi^{-}$invariant mass in the (a) long and (b) downstream datasets, the $K^{+} K^{-}$invariant mass in the (c) long and (d) downstream datasets, and the $p \pi^{-}$invariant mass in the (e) long and (f) downstream datasets. The total fit projection is given by the blue solid line. The blue and green dotted lines represents the $\phi+\Lambda$ and pure combinatorial fit components, respectively. The red and magenta dashed lines represent the $\Lambda_{b}^{0} \rightarrow \Lambda \phi$ signal and the $\Lambda_{b}^{0} \rightarrow \Lambda K^{+} K^{-}$non-resonant components, respectively. Black points represent the data. Data uncertainties are Poisson $68 \%$ confidence intervals. 
calculated to be $5.9 \sigma$, including systematics uncertainties, representing a first observation. The measured branching fraction is $\left(5.18 \pm 1.04 \pm 0.35_{-0.62}^{+0.67}\right) \times 10^{-6}$, where the first uncertainty is statistical, the second is systematic, and the third is related to external inputs. Triple products asymmetries can also be computed in the $\Lambda_{b}^{0} \rightarrow \Lambda \phi$ decay due to the spin structure of the decay. These have been performed following the formalism in [7]. Four triple products are possible, corresponding to $\cos \Phi_{\vec{n}_{\Lambda, \phi}}$ and $\sin \Phi_{\vec{n}_{\Lambda, \phi}}$, where $\Phi_{\vec{n}_{\Lambda, \phi}}$ is the azimuthal angle of the resonance decay planes with normal vector $\vec{n}_{\Lambda, \phi}$. The asymmetries $A_{\Lambda}^{c, s}$ and $A_{\phi}^{c, s}$, where $c$ and $s$ correspond to the cosine and sine respectively, are sensitive to CPV and measured to be $A_{\Lambda}^{c}=-0.22 \pm 0.12$ (stat.) \pm 0.06 (syst.), $A_{\Lambda}^{s}=0.13 \pm 0.12$ (stat.) \pm 0.05 (syst.), $A_{\phi}^{c}=-0.01 \pm 0.12$ (stat.) \pm 0.03 (syst.) and $A_{\phi}^{s}=$ $-0.07 \pm 0.12$ (stat.) \pm 0.01 (syst.), where systematic contributions mainly arise from the mass model. Everything is consistent with zero and no CPV evidence is found.

\section{Study of $\Lambda_{b}^{0} \rightarrow p h^{-} h^{+} h^{-}$decays}

Using data from $\mathrm{LHCb}$ experiment $\mathrm{CPV}$ and $\mathrm{P}$-violating (PV) asymmetries are studied in the decay angle distributions of $\Lambda_{b}^{0} \rightarrow p \pi^{-} \pi^{+} \pi^{-}$and $\Lambda_{b}^{0} \rightarrow p \pi^{-} K^{+} K^{-}$[8]. In this analysis, scalar triple product of final-state particle momenta in the $\Lambda_{b}^{0}$ centre-of-mass frame are studied.These are defined as $C_{\widehat{T}}=\mathbf{p}_{p} \cdot\left(\mathbf{p}_{h_{1}^{-}} \times \mathbf{p}_{h_{2}^{+}}\right)$for $\Lambda_{b}^{0}$ and $\bar{C}_{\widehat{T}}=\mathbf{p}_{\bar{p}} \cdot\left(\mathbf{p}_{h_{1}^{+}} \times \mathbf{p}_{h_{2}^{-}}\right)$for $\bar{\Lambda}_{b}^{0}$, where $h_{1}$ and $h_{2}$ are final-state hadrons: $h_{1}=\pi$ and $h_{2}=K$ for $\Lambda_{b}^{0} \rightarrow p \pi^{-} K^{+} K^{-}$and $h_{1}=h_{2}=\pi$ for $\Lambda_{b}^{0} \rightarrow$ $p \pi^{-} \pi^{+} \pi^{-}$. In the latter case there is an inherent ambiguity in the chioce of the pion for $h_{1}$ that is resolved by taking that with the larger momentum in the $\Lambda_{b}^{0}$ rest frame, referred to as $\pi_{\text {fast }} . A_{\widehat{T}}\left(\bar{A}_{\widehat{T}}\right)$ asymmetry can be defined based on the $C_{\widehat{T}}\left(-\bar{C}_{\widehat{T}}\right)$ sign for $\Lambda_{b}^{0}\left(\bar{\Lambda}_{b}^{0}\right)$. The sum $a_{P}^{\widehat{T} \text {-odd }}=\frac{1}{2}\left(A_{\widehat{T}}+\bar{A}_{\widehat{T}}\right)$ is sensitive to PV while the difference $a_{C P}^{\widehat{T} \text {-odd }}=\frac{1}{2}\left(A_{\widehat{T}}+\bar{A}_{\widehat{T}}\right)$ to CPV effects. The signal yields of $\Lambda_{b}^{0} \rightarrow p \pi^{-} \pi^{+} \pi^{-}$and $\Lambda_{b}^{0} \rightarrow p \pi^{-} K^{+} K^{-}$are 6,646 \pm 105 and 1,030 \pm 56 , respectively, and are extracted from fit shown in Figure 3, where the contribution of background also appear. This is the first observation of these decay modes. We measure the asymmetries
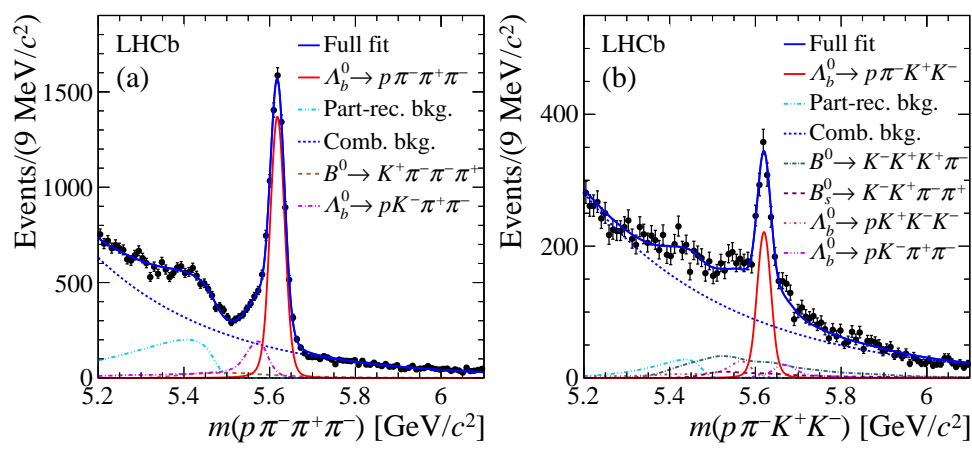

Figure 3: Reconstructed invariant mass fits for (a) $\Lambda_{b}^{0} \rightarrow p \pi^{-} \pi^{+} \pi^{-}$and (b) $\Lambda_{b}^{0} \rightarrow p \pi^{-} K^{+} K^{-}$decays used to extract the signal yields. A fit is overlaid on the top of the data points as a blue line, with red solid (signal) and dotted lines (background) describing the projections of the fit for different contribution.

integrated in phase space obtaining $a_{C P}^{\widehat{T} \text {-odd }}=1.15 \pm 1.45 \pm 0.32(-0.93 \pm 4.54 \pm 0.42)$ and $a_{P}^{\widehat{T} \text {-odd }}=$ $-3.71 \pm 1.45 \pm 0.32(3.62 \pm 4.54 \pm 0.42)$ for $\Lambda_{b}^{0} \rightarrow p \pi^{-} \pi^{+} \pi^{-}\left(\Lambda_{b}^{0} \rightarrow p \pi^{-} K^{+} K^{-}\right)$, consistent with $C P$ and $P$ symmetry hypothesis. The asymmetries are also measured in region of phase space, with 
different binning scheme to isolate regions according to the dominant resonant contributions and to exploits in more detail the interference of contributions which could be visible as a function of the angle $\Phi$ between the decay planes planes formed by the $p \pi_{\text {fast }}^{-}$and the $p \pi_{\text {slow }}^{-}$systems. The combined significance for the two binning schemes for CPV in $\Lambda_{b}^{0} \rightarrow p \pi^{-} \pi^{+} \pi^{-}$decays is determined by means of a permutation test, taking into account correlations among the results. An overall $p$-value of $9.8 \times 10^{-4}(3.3 \sigma)$ is obtained for the $C P$-symmetry hypothesis, including systematic uncertainties. This represents the first evidence for CPV in the baryon sector. The observables measured in phase space for $\Lambda_{b}^{0} \rightarrow p \pi^{-} K^{+} K^{-}$are consistent with $C P$ and $P$ symmetry.

\section{Study of $\Xi_{b}^{-} \rightarrow p h^{-} h^{\prime}-$ decays}

Large CPV effects have been observed in regions of the phase space of $B^{-} \rightarrow h^{+} h^{\prime} h^{\prime \prime}$ mesonic decays [9-11]. The baryonic partner is represented by $\Xi_{b}^{-}\left(\Omega_{b}^{-}\right) \rightarrow p h^{-} h^{\prime}-$ and it is therefore of great interest to search for these charmless $b$-baryon decays that may be used to investigate CPV effects. For the first time these decays are searched with the LHCb detector [12]. The result of the fit after the selection is shown in Figure 4. The decay $\Xi_{b}^{-} \rightarrow p K^{-} K^{-}$is observed with a significance
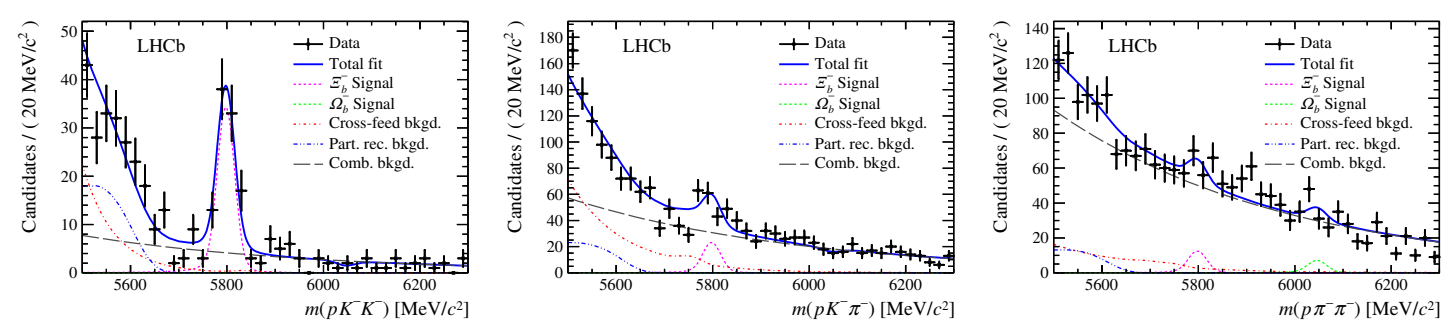

Figure 4: Mass distributions for $b$-hadron candidates in the (left) $p K^{-} K^{-}$, (center) $p K^{-} \pi^{-}$, (right) $p \pi^{-} \pi^{-}$. Results of the fits are shown with dark blue solid lines. Signals for $\Xi_{b}^{-}\left(\Omega_{b}^{-}\right)$decays are shown with pink (light green) dashed lines, combinatorial backgrounds are shown with grey long-dashed lines, cross-feed backgrounds are shown with red dot-dashed lines, and partially reconstructed background are shown with dark blue double-dot-dashed lines.

of $8.7 \sigma$, and evidence at the level of $3.4 \sigma$ is found for the $\Xi_{b}^{-} \rightarrow p K^{-} \pi^{-}$decay. This is the first observation of a $\Xi_{b}$ decay to a charmless final state. The measured branching ratio are

$$
\begin{gathered}
\frac{f_{\Xi_{b}^{-}}}{f_{u}} \frac{\mathscr{B}\left(\Xi_{b}^{-} \rightarrow p K^{-} K^{-}\right)}{\mathscr{B}\left(B^{-} \rightarrow K^{+} K^{-} K^{-}\right)}=(2.65 \pm 0.35 \pm 0.47) \times 10^{-3}, \\
\frac{\mathscr{B}\left(\Xi_{b}^{-} \rightarrow p K^{-} \pi^{-}\right)}{\mathscr{B}\left(\Xi_{b}^{-} \rightarrow p K^{-} K^{-}\right)}=0.98 \pm 0.27 \pm 0.09
\end{gathered}
$$

where the decay $\Xi_{b}^{-} \rightarrow p K^{-} K^{-}$is measured with respect to the control sample $B^{-} \rightarrow K^{+} K^{-} K^{-}$. All the other $\Xi_{b}^{-}$and $\Omega_{b}^{-}$signals are not statistically significant. For those modes an upper limit at 90 (95)\% CL is given

$$
\begin{array}{ll}
\frac{f_{\Xi_{b}^{-}}}{f_{u}} \frac{\mathscr{B}\left(\Xi_{b}^{-} \rightarrow p \pi^{-} \pi^{-}\right)}{\mathscr{B}\left(B^{-} \rightarrow K^{+} K^{-} K^{-}\right)}<1.47(16.6) \times 10^{-4}, & \frac{f_{\Omega_{b}^{-}}}{f_{u}} \frac{\mathscr{B}\left(\Omega_{b}^{-} \rightarrow p K^{-} K^{-}\right)}{\mathscr{B}\left(B^{-} \rightarrow K^{+} K^{-} K^{-}\right)}<1.8(2.2) \times 10^{-4}, \\
\frac{f_{\Omega_{b}^{-}}}{f_{u}} \frac{\mathscr{B}\left(\Omega_{b}^{-} \rightarrow p K^{-} \pi^{-}\right)}{\mathscr{B}\left(B^{-} \rightarrow K^{+} K^{-} K^{-}\right)}<5.1(6.2) \times 10^{-4}, & \frac{f_{\Omega_{b}^{-}}}{f_{u}} \frac{\mathscr{B}\left(\Omega_{b}^{-} \rightarrow p \pi^{-} \pi^{-}\right)}{\mathscr{B}\left(B^{-} \rightarrow K^{+} K^{-} K^{-}\right)}<10.9(12.4) \times 10^{-4} .
\end{array}
$$




\section{Conclusions}

Four recent results from $\mathrm{LHCb}$ in the field of charmless $b$-baryon decays have been presented. The decay modes $\Lambda_{b}^{0} \rightarrow \Lambda K^{+} K^{-}, \Lambda_{b}^{0} \rightarrow \Lambda K^{+} \pi^{-}$and $\Lambda_{b}^{0} \rightarrow \Lambda \phi$ are observed for the first time and their branching fraction is measured. The phase-space integrated $C P$ asymmetry parameters are measured for the $\Lambda_{b}^{0} \rightarrow \Lambda K^{+} K^{-}$and $\Lambda_{b}^{0} \rightarrow \Lambda K^{+} \pi^{-}$decays, while several triple-product asymmetries are measured for the $\Lambda_{b}^{0} \rightarrow \Lambda \phi$ decay. All these asymmetries are found to be compatible with zero. Triple-product asymmetries are measured for $\Lambda_{b}^{0} \rightarrow p \pi^{-} K^{+} K^{-}$and $\Lambda_{b}^{0} \rightarrow p \pi^{-} \pi^{+} \pi^{-}$decays, observed for the first time, both integrated in phase space and in region of phase space. A deviation at $3.3 \sigma$ from the $C P$ symmetry hypothesis is found in regions of phase space for $\Lambda_{b}^{0} \rightarrow p \pi^{-} \pi^{+} \pi^{-}$decay. This represents the first evidence of CPV in baryon decays. All the other measured asymmetries don't show any deviation. The first observation of $\Xi_{b}^{-} \rightarrow p K^{-} K^{-}$decay and the first evidence for $\Xi_{b}^{-} \rightarrow p K^{-} \pi^{-}$decay have been obtained. This is the first observation of a $\Xi_{b}$ decay to a charmless final state. These modes may be used in future to search for $C P$ asymmetries in the $b$-baryon sector.

\section{References}

[1] LHCb collaboration, Determination of the quark coupling strength $\left|V_{u b}\right|$ using baryonic decays, Nature Phys. 11743 (2015) [hep-ex/1504.01568].

[2] LHCb collaboration, Observation of $J / \psi p$ Resonances Consistent with Pentaquark States in $\Lambda_{b}^{0} \rightarrow J / \psi K^{-}$p Decays, Phys. Rev. Lett. 115072001 (2015) [hep-ex/1507.03414].

[3] LHCb collaboration, Observation of two new $\Xi_{b}^{-}$baryon resonances, Phys. Rev. Lett. 114062004 (2015) [hep-ex/1411.4849].

[4] LHCb collaboration, Observation of excited $\Lambda_{b}^{0}$ baryons, Phys. Rev. Lett. 109172003 (2012) [hep-ex/1205.3452].

[5] LHCb collaboration, Observations of $\Lambda_{b}^{0} \rightarrow \Lambda K^{+} \pi^{-}$and $\Lambda_{b}^{0} \rightarrow \Lambda K^{+} K^{-}$decays and searches for other $\Lambda_{b}^{0}$ and $\Xi_{b}^{0}$ decays to $\Lambda h^{+} h^{\prime-}$ final states, JHEP 05081 (2016) [hep-ex/1603.00413].

[6] LHCb collaboration, Observation of the $\Lambda_{b}^{0} \rightarrow \Lambda \phi$ decay, Phys. Lett. $\mathbf{B 7 5 9} 282$ (2016) [hep-ex/1603.02870].

[7] Leitner, O. and Ajaltouni, Z. J., Testing CP and Time Reversal Symmetries with $\Lambda_{b}^{0} \rightarrow \Lambda V(1-)$ Decays, Nucl. Phys. Proc. Suppl. 174169 (2007) [hep-ph/ 0610189$].$

[8] LHCb collaboration, Measurement of matter-antimatter differences in beauty baryon decays, Nature Phys. Online publication (2017) [hep-ex/1609.05216].

[9] LHCb collaboration, Measurement of CP violation in the phase space of $B^{ \pm} \rightarrow K^{ \pm} \pi^{+} \pi^{-}$and $B^{ \pm} \rightarrow K^{ \pm} K^{+} K^{-}$decays, Phys. Rev. Lett. 111101801 (2013) [hep-ex/1306.1246].

[10] LHCb collaboration, Measurement of CP violation in the phase space of $B^{ \pm} \rightarrow K^{+} K^{-} \pi^{ \pm}$and $B^{ \pm} \rightarrow \pi^{+} \pi^{-} \pi^{ \pm}$decays, Phys. Rev. Lett. 112011801 (2014) [hep-ex/1310.4740].

[11] LHCb collaboration, Measurements of CP violation in the three-body phase space of charmless $B^{ \pm}$ decays, Phys. Rev. D90 112004 (2014) [hep-ex/1408.5373].

[12] LHCb collaboration, Observation of the decay $\Xi_{b}^{-} \rightarrow p K^{-} K^{-}$, Phys. Rev. Lett. 118071801 (2017) [hep-ex/1612.02244]. 